\title{
Combined Failure Diagnosis of Slewing Bearings Based on MCKD-CEEMD-ApEn
}

\author{
Fengtao Wang $\mathbb{D}^{1},{ }^{1}$ Chenxi Liu $\mathbb{D},{ }^{1}$ Wensheng Su $\mathbb{D},{ }^{2}$ Zhigang Xue $\mathbb{D}{ }^{2}{ }^{2}$ \\ Qingkai Han (D), ${ }^{1}$ and Hongkun Li ${ }^{1}{ }^{1}$ \\ ${ }^{1}$ Institute of Vibration Engineering, Dalian University of Technology, Dalian 116024, China \\ ${ }^{2}$ Jiangsu Province Special Equipment Safety Supervision Inspection Institute, Branch of Wuxi, Wuxi 214071, China \\ Correspondence should be addressed to Fengtao Wang; wangft@dlut.edu.cn
}

Received 31 January 2018; Revised 12 March 2018; Accepted 15 March 2018; Published 23 April 2018

Academic Editor: Carlo Rainieri

Copyright (C) 2018 Fengtao Wang et al. This is an open access article distributed under the Creative Commons Attribution License, which permits unrestricted use, distribution, and reproduction in any medium, provided the original work is properly cited.

Large-size and heavy-load slewing bearings, which are mainly used in heavy equipment, comprise a subgroup of rolling bearings. Owing to the complexity of the structures and working conditions, it is quite challenging to effectively diagnose the combined failure and extract fault features of slewing bearings. In this study, a method was proposed to denoise and classify the combined failure of slewing bearings. First, after removing the mean, the vibration signals were denoised by maximum correlated kurtosis deconvolution. The signals were then decomposed into several intrinsic mode functions (IMFs) by complementary ensemble empirical mode decomposition (CEEMD). Appropriate IMFs were selected based on the correlation coefficient and kurtosis. The approximate entropy values of the selected IMFs were regarded as the characteristic vectors and then inputted into the support vector machine (SVM) based on multiclass classification for training. The practical combined failure signals of the 3 conditions were finally recognized and classified using SVMs. The study also compared the proposed method with 5 other methods to demonstrate the superiority and effectiveness of the proposed method.

\section{Introduction}

Large-size and heavy-load slewing bearings are widely used in major and heavy equipment. Faults more easily occur in slewing bearings, compared with other machine parts under practical working conditions [1]. Owing to the strong background noise interference, extracting combined fault features from vibration signals has long been the focus of current studies. A common approach is to diagnose the faults of time-domain vibration signals after the denoising process [2-5], aiming to increase the signal-to-noise ratio (SNR) and reduce the noise interference.

The maximum correlated kurtosis deconvolution (MCKD) algorithm was first proposed by McDonald et al. [6]. This method can increase the correlated kurtosis values of the signals as well as the SNR of the weak fault signals by deconvolution. Meanwhile, the submerged successive pulses can be given emphasis. Compared with the minimum entropy deconvolution [7] algorithm, the MCKD algorithm can also eliminate strong background noise interference to achieve predenoising. The MCKD algorithm is currently applied in many fault diagnosis studies on large-size rolling bearings. Jia et al. [8] combined MCKD with improved spectrum kurtosis to diagnose vibration signals and extract the fault features of rolling bearings in wind turbines and hot-strip rolling mills. Zhao and Li [9] used MCKD and empirical mode decomposition (EMD) method to extract the early-stage fault features of rolling bearings in wind turbines from strong background noise. The present study focused on the denoising effect of the MCKD algorithm and the time-domain analysis of the vibration signals. Thus, the study of frequency spectrum was not demonstrated.

Complementary ensemble empirical mode decomposition (CEEMD) [10] was proposed based on ensemble empirical mode decomposition (EEMD) [11]. In CEEMD, a pair of opposite white noises is added to the original signals first. The recombined signals are then decomposed by EMD to obtain a series of intrinsic mode functions 
(IMFs). Compared with EEMD, CEEMD can reduce the reconstruction error of the added white noise as well as mode mixing under the same decomposition effects. As a signal decomposition method, CEEMD is typically combined with other techniques to diagnose faults in machines [12-14]. In our study, CEEMD was used to denoise signals and extract characteristic parameters for further fault classification.

Currently, some research achievements on the combination of signal decomposition methods and many kinds of entropy have been published to apply on the fault diagnosis and classification of rolling bearings. Entropy is a nonlinearity index for evaluating the irregularity of signals. As a category of significant phase-space indexes, many kinds of entropy were used as characteristic parameters in fault diagnosis and classification of rolling bearings [15-18]. Approximate entropy (ApEn), proposed by Pincus [19], can quantize vibration signals and achieve feature extraction, which exhibits strong anti-interference. The more complex the time series, the bigger the ApEn value. ApEn has been considered in recent years as an effective characteristic parameter in condition monitoring and fault diagnosis [2022]. Caesarendra et al. [23] analyzed four nonlinear features, including ApEn, largest Lyapunov exponent (LLE), and correlation dimension (CD), to provide more superior descriptive information about slewing bearings than timedomain features. Although most studies focused on a single artificial fault, this study focused on practical combined faults under different conditions. This study used ApEn as the characteristic parameter to diagnose the faults and extract the features of large-size and heavy-load slewing bearings under various working conditions. For comparison, sample entropy (SE), LLE, and CD were also taken as the characteristic parameters to justify the benefits of ApEn in this study.

Support vector machine (SVM) is a kind of learning algorithm proposed by Vapnik [24] in 1998. In a classification process, SVM separates a set of labeled training data with a hyperplane that maximizes its distance to the data. So, the SVM can work in combination with kernel functions to compute a nonlinear mapping to the features space. The hyperplane corresponds to a nonlinear decision boundary in the input space [25]. SVM has strong function fitting advantages and it is suited for resolving small-sample and nonlinear model identification. Compared with other intelligent classifiers, this one requires a smaller sample size to seek for the optimal solution based on finite samples, which can be taken as a convex optimization problem. The existence and acquisition of the globally optimal solution can be guaranteed. With sound robustness, the empirical risk and confidence risk are taken into consideration in SVM for resolving small-sample problems. The optimal solution based on finite information rather than infinite samples is acquired. In terms of popularization, SVM is more appropriate to be used in fault diagnosis of small-sample conditions. Xiong et al. [26] developed a scheme using SVMs for diagnosing bearing conditions, where a $97.42 \%$ accuracy is achieved. Zhang et al. [27] proposed a fault diagnosis scheme for locomotive roller bearings using an SVM classifier. Compared with some methods based on other neural networks, SVM has both a simple structure and an improved generalization capability.
The original vibration signals were denoised by removing the mean and the MCKD algorithm as well as conducting decomposition by CEEMD to obtain IMFs. The selected IMFs, which could represent the features of the original signals, were extracted ApEn values. The values consisted of characteristic vectors and were then inputted into the SVMs to train in order to identify the faults of the slewing bearings. All the vibration data of the slewing bearings applied in this study resulted from the practical working conditions. The remaining sections of this paper are arranged as follows. The theories supporting the proposed method are presented in Section 2 . The practical signal verification conducted on the slewing bearing of a practical port crane is demonstrated in Section 3. The last section provides the summary and conclusions of the study.

\section{Theoretical Framework}

2.1. Maximum Correlated Kurtosis Deconvolution. MCKD uses correlated kurtosis as its index and emphasizes periodic pulse components submerged in the background noise by deconvolution computation. Iteration ends when the correlated kurtosis values of the original signals reach the peak. The algorithm achieves denoising for the impact failure of rolling bearings and highlights shock pulses. The vibration signal when faults occur in a rolling bearing can be described as follows:

$$
y(n)=h(n) * x(n)+e(n) .
$$

The MCKD algorithm aims to determine the FIR filter $w(l)$, which can achieve the maximal correlated kurtosis value of the original signal. The input signal $x(n)$ can be obtained using the output signal $y(n)$ using the following:

$$
x(n)=w(n) * y(n) .
$$

The correlated kurtosis of the MCKD algorithm is defined as

$$
\mathrm{CK}_{M}(T)=\frac{\sum_{n=1}^{N}\left(\prod_{m=0}^{M} y(n-m T)\right)^{2}}{\left(\sum_{n=1}^{N} y^{2}(n)\right)^{M+1}},
$$

where $T$ represents the period of the impulse signal and $M$ denotes the shift order. The higher the value of $M$, the greater the impulse sequences of deconvolution, which indicates that the fault-detecting ability is stronger. The optimized objective function of MCKD can be described as follows:

$$
\operatorname{MCKD}_{M}(T)=\max _{w(l)} \frac{\sum_{n=1}^{N}\left(\prod_{m=0}^{M} y_{n-m T}\right)^{2}}{\left(\sum_{n=1}^{N} y_{n}^{2}\right)^{M+1}},
$$

where $l=1,2, \ldots, L$. The optimized filter $w(l)$ should satisfy the equation

$$
\frac{\mathrm{d}}{\mathrm{d} w(l)} \mathrm{CK}_{m}(T)=0 .
$$

The results of $w(l)$ can be described in the form of a matrix as follows:

$$
w=\frac{\left\|\mathbf{y}^{2}\right\|}{2\|\mathbf{B}\|^{2}}\left(\mathbf{X}_{\mathbf{0}} \mathbf{X}_{\mathbf{0}}^{\mathbf{T}}\right)^{-1} \sum_{m=0}^{M} \mathbf{X}_{m \mathbf{T}} \mathbf{A}_{m}
$$


where

$$
\begin{aligned}
& \mathbf{X}_{r}=\left[\begin{array}{cccc}
x_{1-r} & x_{2-r} & \cdots & x_{N-r} \\
0 & x_{1-r} & \cdots & x_{N-1-R} \\
\vdots & \vdots & \vdots & \vdots \\
0 & 0 & \cdots & x_{N-L-r+1}
\end{array}\right]_{L \times N} \\
& r=\left[\begin{array}{lllll}
0 & T & 2 T & \cdots & m T
\end{array}\right], \\
& \mathbf{A}_{m}=\left[\begin{array}{ccc}
y_{1-m T}^{-1}\left(y_{1}^{2} y_{1-T}^{2}\right. & \cdots & \left.y_{1-m T}^{2}\right) \\
\vdots & & \\
y_{N-m T}^{-1}\left(y_{N}^{2} y_{N-T}^{2}\right. & \cdots & \left.y_{N-m T}^{2}\right)
\end{array}\right]_{N \times 1}, \\
& \mathbf{B}=\left[\begin{array}{ccc}
y_{1} y_{1-T} & \cdots & y_{1-M T} \\
& \vdots & \\
y_{N} y_{N-T} & \cdots & y_{N-M T}
\end{array}\right]_{N \times 1} \text {. }
\end{aligned}
$$

Finally, the coefficient inputs are incorporated into (2) to obtain $x(n)$. This study used MCKD for signal denoising. Different parameters affected the result of denoising. In general, the shift order is selected from 1 to 7 [28]. Although a higher value can obtain more successive pulses, the period of the impulse signal should be estimated more accurately; in addition, the computational cost can increase. The practical fault frequency also varies from the theoretical result. With all factors considered, the shift order selected is 4 . The filter size is another important parameter. This study determined the sizes as the kurtosis values of the vibration signals from different working conditions reaching their maximum.

\subsection{Complementary Ensemble Empirical Mode Decomposi-} tion. As a signal decomposition method, CEEMD adds a pair of Gaussian white noises with opposite signs, which can not only ensure the continuity of signals but also completely neutralize the added noise. First, $I$ power white noise is added in the original signal $x(n), i=1,2, \ldots, I$. The numbers of white noises are added in pairs to obtain $2 I$ signals:

$$
\left[\begin{array}{l}
x_{+} \\
x_{-}
\end{array}\right]=\left[\begin{array}{cc}
1 & 1 \\
1 & -1
\end{array}\right]\left[\begin{array}{l}
x \\
\omega_{i}
\end{array}\right] \text {. }
$$

Each signal $x^{i}(n)$ in the set is decomposed by EMD; a group of $\operatorname{IMF}_{k}^{i}(n)$ can then be obtained. The first one can be described as $\operatorname{IMF}_{1}(n)=(1 / 2 I) \sum_{i=1}^{2 I} \operatorname{IMF}_{k}^{i}(n)$ and the first residual error can be described as $r_{1}(n)=x(n)-\operatorname{IMF}_{1}(n)$. As $k=2,3, \ldots, K$, the $k$ th residual error can be calculated as follows:

$$
r_{k}(n)=r_{k-1}(n)-\mathrm{IMF}_{k}(n)
$$

The decomposition function becomes $r_{k}(n)+\xi_{k} E_{k}\left(\omega_{i}(n)\right)$ :

$$
\operatorname{IMF}_{k+1}(n)=\frac{1}{2 I} \sum_{i=1}^{2 I} E_{1}\left(r_{k}+\xi_{k} E_{k}\left(\omega_{i}(n)\right)\right),
$$

where $\xi_{k}$ represents SNR and $E_{j}(\cdot)$ denotes the $j$ th decomposition function after EMD. The aforementioned steps are repeated until the selection is ended. The signal can ultimately be described as follows:

$$
x(n)=\sum_{k=1}^{k} \operatorname{IMF}(n)_{k}+R(n) .
$$

We referred to the study by Huang and $\mathrm{Wu}$ [29] to decrease the error caused by the residual noise below $1 \%$, and the amplitude of white noise was set to $1 / 10$ th of the original signal.

2.3. Approximate Entropy. ApEn can be used to quantify the vibration signals on the basis of the complexity of its information, which can be applied in feature extraction. As for the time series $\{x(1), x(x), \ldots, x(N)\}$, which contains $N$ data, the ApEn algorithm predetermines the mode dimension $m$ to reconstruct the phase space. The elements in the time series are extracted to form an $m$ - $D$ vector:

$$
\mathbf{X}(i)=[x(i), x(i+1), \ldots, x(i+m-1)] .
$$

The maximum difference between $\mathbf{X}(i)$ and $\mathbf{X}(j)$ is defined as the distance:

$$
d[\mathbf{X}(i), \mathbf{X}(j)]=\max _{k=0,1, \ldots, m-1}\{|x(i+k)-x(j+k)|\} .
$$

On the basis of the predetermined threshold value $r$, the number of distances lower than $r$ accumulates:

$$
C_{i}^{m}(r)=\frac{1}{N-m+1} \operatorname{Num}\{d[\mathbf{X}(i), \mathbf{X}(j)]<r\},
$$

where $i, j=1,2, \ldots, N-m+1, i \neq j . C_{i}^{m}(r)$ represents the degree of correlation between $\mathbf{X}(i)$ and $\mathbf{X}(j)$. $\Phi^{m}(r)$ denotes the degree of autocorrelation of $\left\{\mathbf{X}_{i}\right\}$ :

$$
\Phi^{m}(r)=\frac{1}{N-m+1} \sum_{i=1}^{N-m+1} \ln C_{i}^{m}(r) .
$$

The mode dimension is expanded from $m$ to $m+1$. $\Phi^{m+1}(r)$ can be obtained after the repetition of the aforementioned steps. The ApEn value of the time series can be obtained based on $\Phi^{m}(r)$ and $\Phi^{m+1}(r)$ :

$$
\operatorname{ApEn}(m, r)=\lim _{N \rightarrow \infty}\left[\Phi^{m}(r)-\Phi^{m+1}(r)\right]
$$

This study sets ApEn as the feature parameter. To obtain improved statistical property and reduce error, the threshold value was set to $1 / 10$ th of the standard deviation [19].

2.4. Support Vector Machines. In order to resolve the 3classification problem in this study, one-against-one method is adopted, in which SVMs are constructed for all possible pairs of classes including (I) normal condition-early fault condition (SVM1), (II) normal condition-severe fault condition (SVM2), and (III) early fault condition-severe fault condition (SVM3). The decision function that separates the 


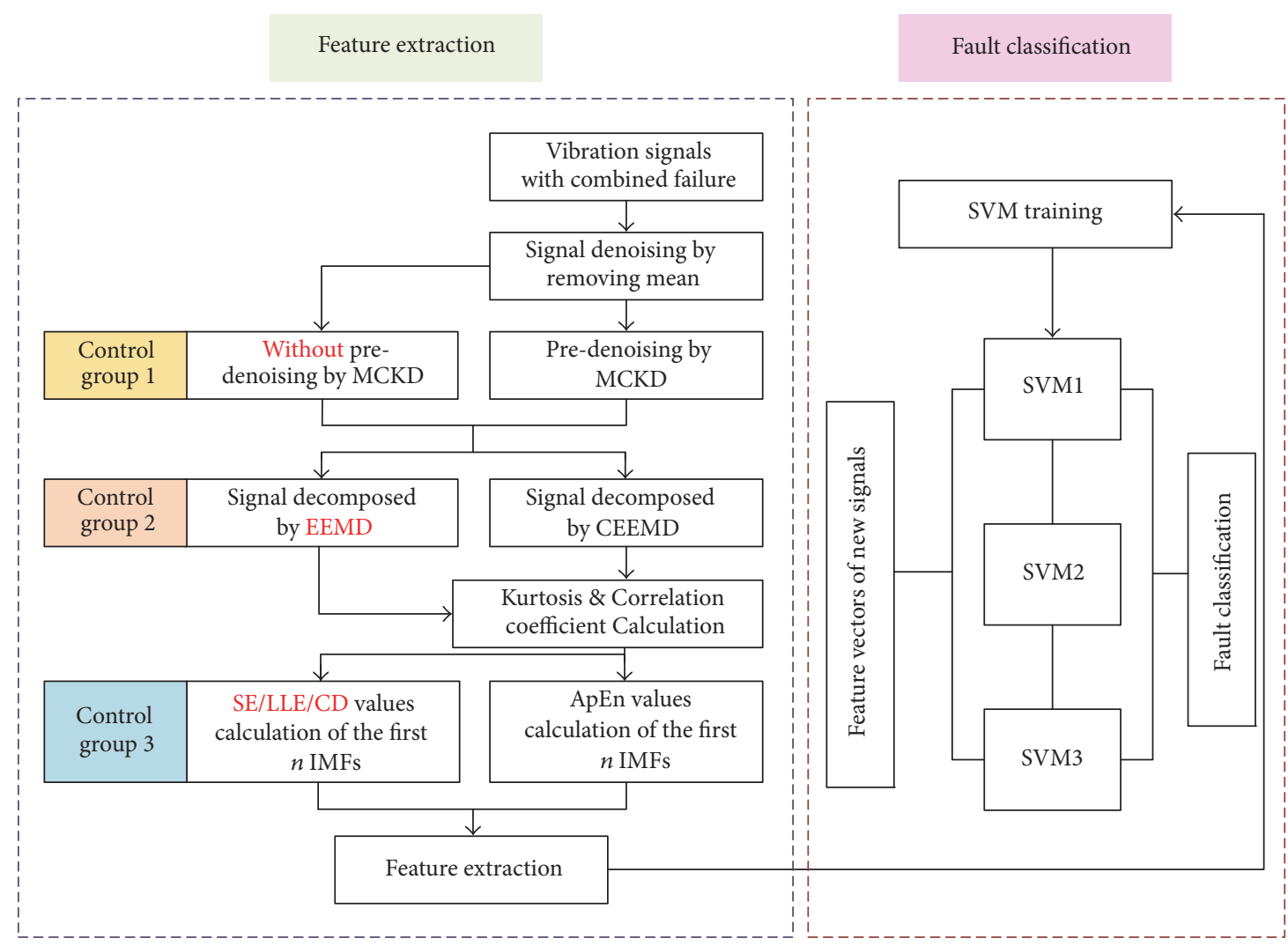

FIGURE 1: Flow chart of the proposed method.

$i$ th from the $j$ th class for the features vector is defined as follows:

$$
d_{i j}(\mathbf{x}) \triangleq \mathbf{w}_{i j}^{T} \mathbf{x}+b_{i j},
$$

where $i, j=1,2, \ldots, N, \mathbf{w}_{i j} \in \mathbf{R}^{n}$ is a weight vector, $\mathbf{x}_{i} \in \mathbf{R}^{n}$ is the $i$ th features sample, and $b_{i j}$ is a bias term. The number of times that the features vector $\mathbf{x}$ is assigned to the $i$ th class is computed by

$$
d_{i}(\mathbf{x})=\sum_{\substack{j=1 \\ j \neq i}}^{N} \operatorname{sign}\left(d_{i j}(\mathbf{x})\right) .
$$

The following decision function is considered:

$$
d(\mathbf{x})=\arg \max _{i=1,2, \ldots, N} d_{i}(\mathbf{x}) .
$$

The highest number of votes that a feature $\mathbf{x}$ vector may have is $N-1$. For the design of the SVMs, the Gaussian RBF kernel is considered:

$$
K\left(\mathbf{x}_{i}, \mathbf{x}_{j}\right)=\exp \left(-\frac{\left\|\mathbf{x}_{i}-\mathbf{x}_{j}\right\|^{2}}{2 \sigma^{2}}\right),
$$

where $\left\|\mathbf{x}_{i}-\mathbf{x}_{j}\right\|$ denotes the Euclidean norm and $\sigma$ is a free parameter related to the dispersion of the support vectors. RBF functions have been documented extensively in the literature, making them a benchmark for classification applications based on SVMs [25].
2.5. Fault Diagnosis Based on MCKD-CEEMD-ApEn. Figure 1 demonstrates the specific implementation steps of the proposed method.

Step 1. Remove the mean of the original signals and predenoise the signals by MCKD.

Step 2. Decompose the denoised signals by CEEMD and select appropriate IMFs based on kurtosis and the correlation coefficient.

Step 3. Calculate the ApEn values of each selected IMF and compose the characteristic vectors.

Step 4. Input the characteristic vectors into the SVM for training and verify the fault classification rate and validity of the proposed method.

\section{Experimental Evaluation}

All the time-domain signals used in this study are selected from the large-size, heavy-load slewing bearing of a practical port crane. The type of the slewing bearing type was 132.45.2800.03. The slewing bearing began to serve from Jul. 2013 and its vibration data were collected from Feb. 2015 to Dec. 2017 about every 2 months. No artificial defect was manufactured on the slewing bearing for fault simulation. The complicated practical working condition and equipment led to combined faults occurring on the slewing bearing. In 


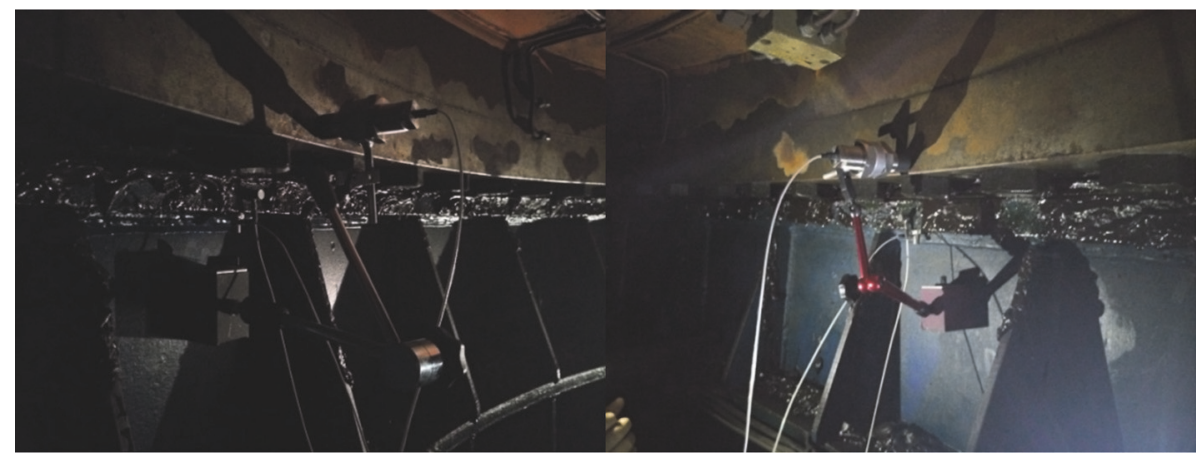

Figure 2: Arrangement of sensors.

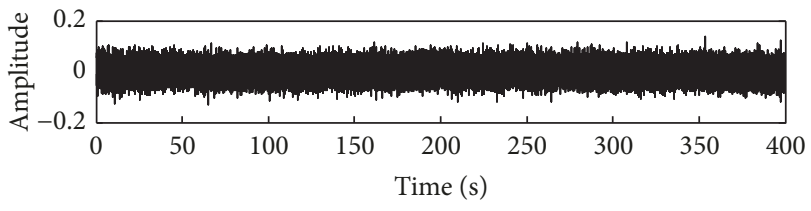

(a) Normal condition

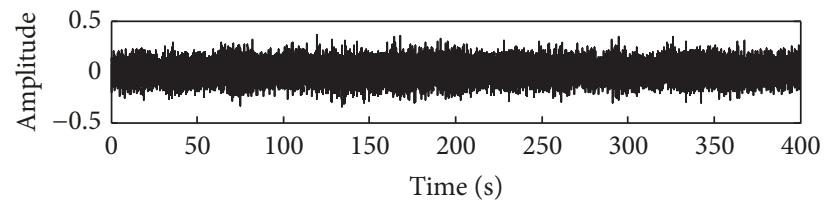

(b) Early fault condition

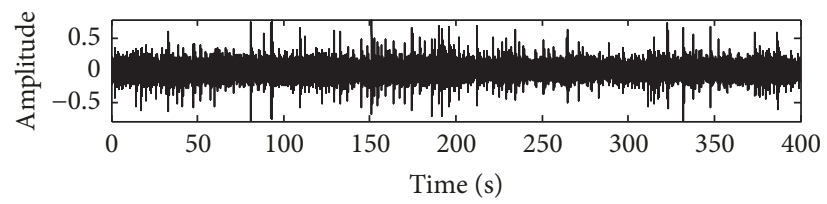

(c) Severe fault condition

FIGURE 3: Time-domain signals of various conditions (the data were acquired on Oct. 15, 2015; Oct. 25, 2016; and Oct. 26, 2017, resp.).

order to reduce human interference as much as possible, the outer ring, inner ring, rolling elements, and gear teeth of the slewing bearing did not, on the condition of operational safety, undergo any maintenance except for a disassembly check every 6 to 7 months and regular lubrication. Except for the periods of data collection, the slewing bearing maintained normal operation process. For analysis convenience, during the periods of data collection, the rotating speed of the inner race was $1.5 \mathrm{rpm}$ and the rotation direction was counterclockwise. The container carried by the crane remained the same each time. Each acquisition duration of the vibration signals collected using PCB acceleration sensors was longer than a rotation cycle. The sensors were placed on the radial surface at a $90^{\circ}$ angle relative to one another around the ring (Figure 2 ). As shown in Figure 3, the vibration signals under 3 different conditions were collected: those under the normal condition, early fault condition, and severe fault condition. The sampling frequency was $512 \mathrm{~Hz}$. To decrease the calculated amount and for ease of comparison, a one-rotation-cycle (40s) vibration signal was acquired each time. A single fault rarely occurs in practical working slewing bearings, unlike the theoretical analysis as well as the experimental simulation. In addition, according to the disassembly check records (Figure 4), combined faults at varying degrees were observed in the slewing bearing in the early fault and severe fault stages. The working conditions of the slewing bearing were divided into 3 stages based on the deterioration degree of the combined failure.
In the normal working stage (from Feb. 2015 to Jun. 2016), the whole bearing was complete, and all components were of normal size. No obvious pitting or abrasion occurred; the lubrication was sound, and the noise was weak. In the early fault stage (from Jul. 2016 to Aug. 2017), large-scale pitting or abrasion occurred in the outer race and the inner race of the slewing bearing. In addition, some rolling elements, retainers, and gear teeth were damaged. Several metal particles could be found in the lubricating grease. In the severe fault stage (from Sep. 2017 to Dec. 2017), abrasion occurred in most rolling elements of the three rows. More serious damage, abrasion, and cracks occurred in the outer gear teeth, some of which were broken. The effects were apparently accompanied by strong noise. Many metal impurities could be found in the lubricating grease. Thus, the combined failure could be determined in the early and severe fault stages.

To verify the superiority and validity of the proposed method, 3 control methods were conducted in this study as follows:

(1) Decompose the signals by CEEMD directly after removing the mean without predenoising by MCKD.

(2) Decompose the signals by EEMD rather than CEEMD after MCKD.

(3) Use SE/LLE/CD, rather than ApEn, as the characteristic indexes. 


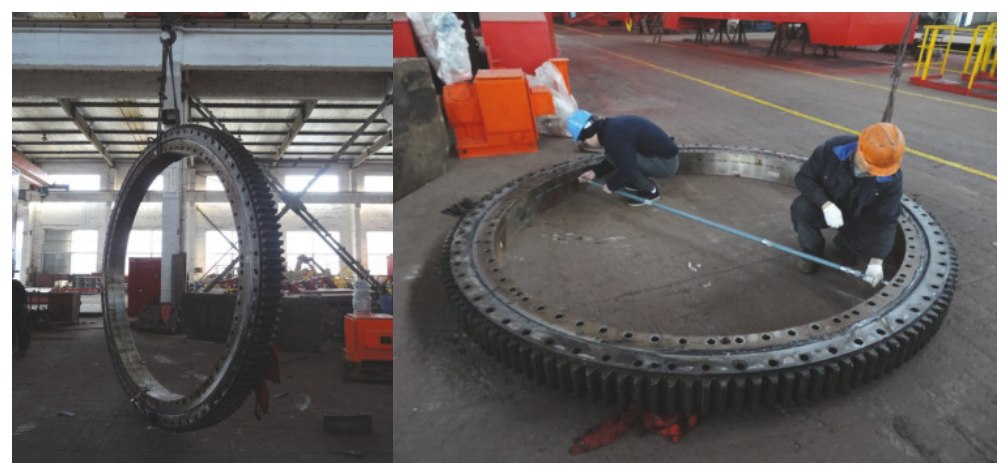

(A) Normal condition

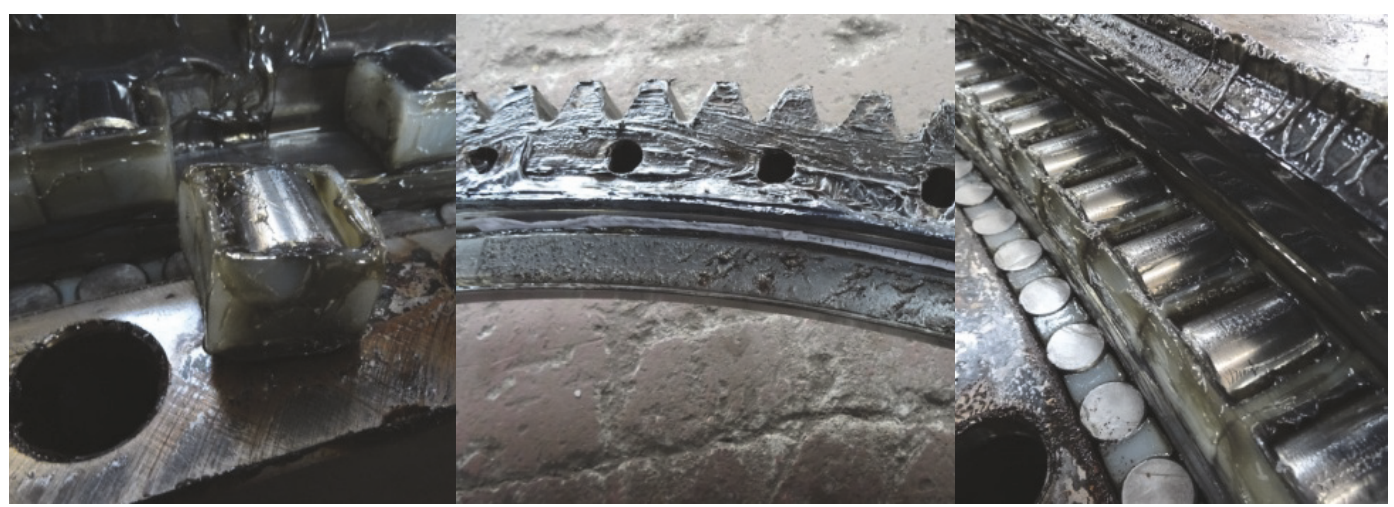

(B) Early fault condition

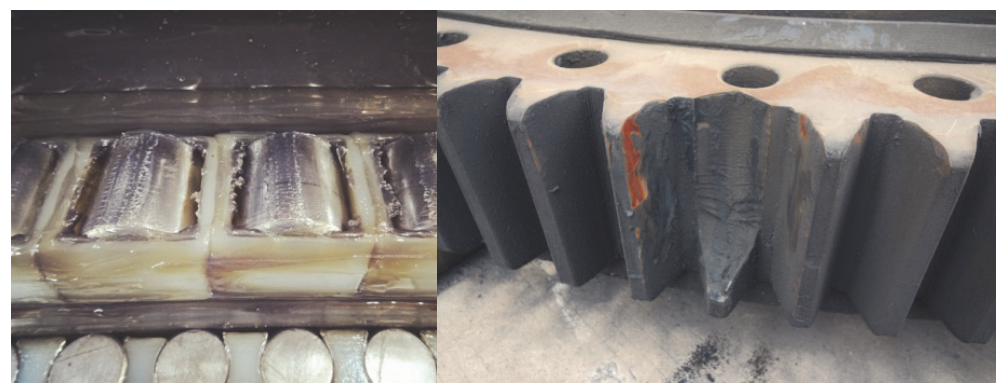

(C) Severe fault condition

FIGURE 4: Faults of various conditions.

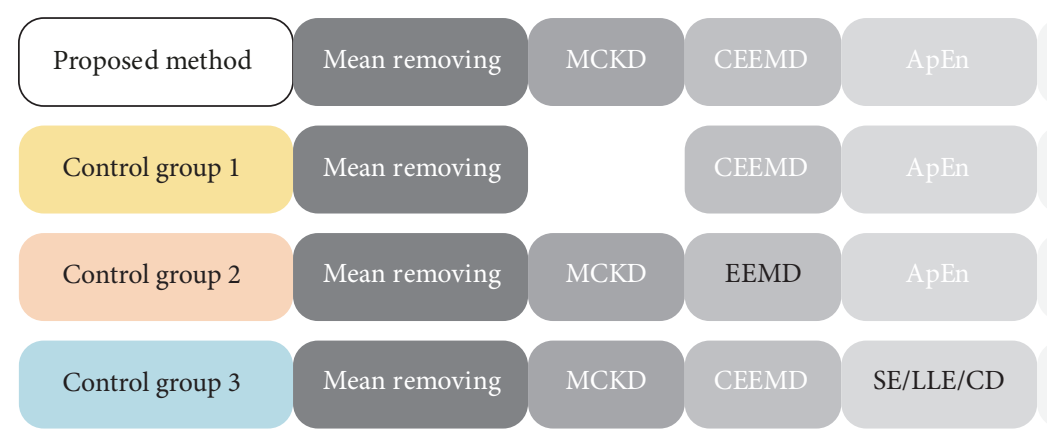

Figure 5: Methods comparison. 


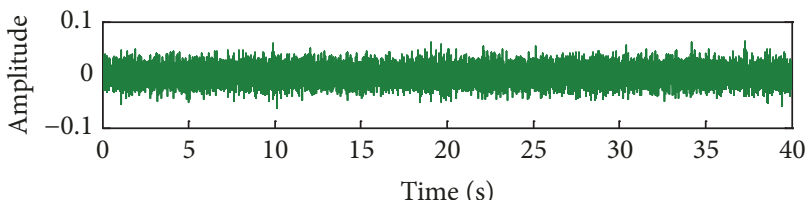

(a) Normal condition

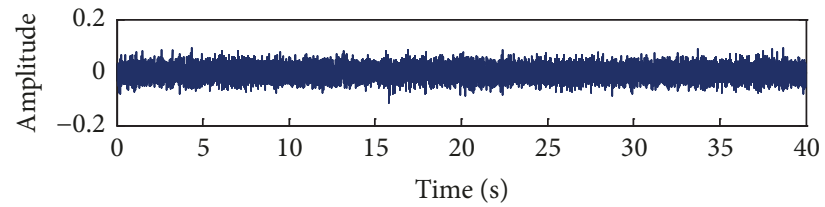

(b) Early fault condition

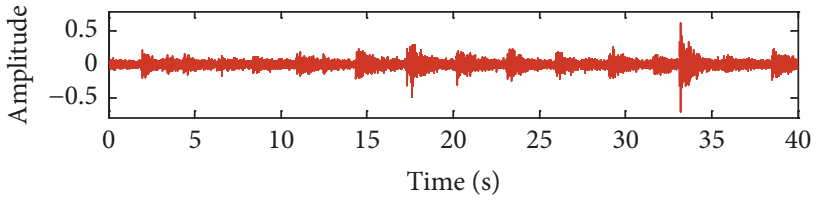

(c) Severe fault condition

FIGURE 6: Time-domain signals of various conditions after MCKD (the data were acquired on Oct. 15, 2015; Oct. 25, 2016; and Oct. 26, 2017, resp.).

\section{$\sum_{1}$}

\section{$\sum_{i}^{N}$}
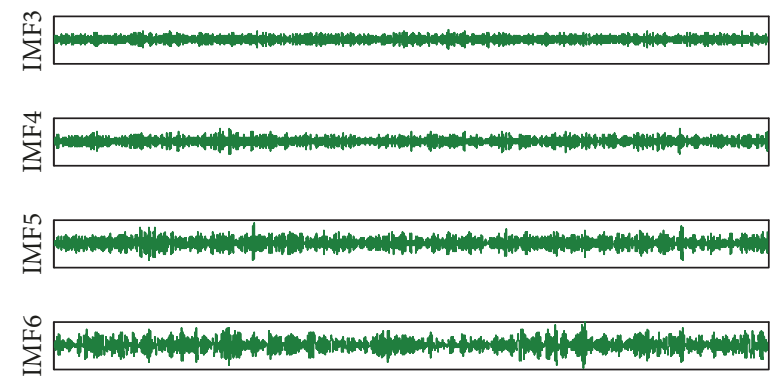

尊

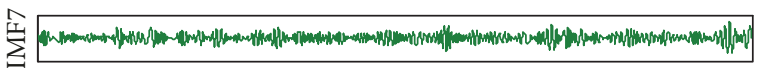

$\sum_{i=1}^{\infty}$

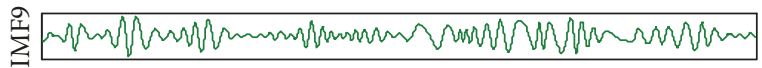

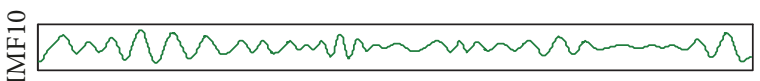
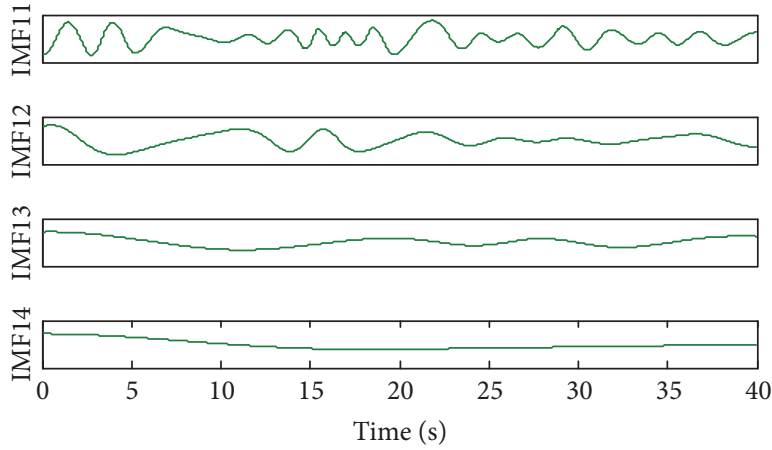

(a) CEEMD
牙

$\sum_{i=1}^{N}$

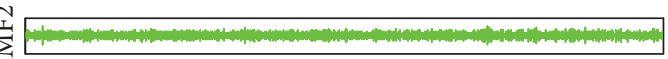

$\sum^{M}$

$\sum_{2}^{+}$

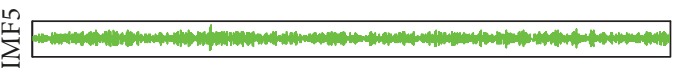

$\sum_{i}^{0}$

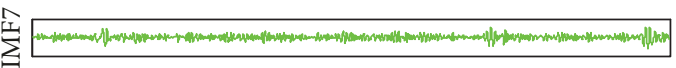

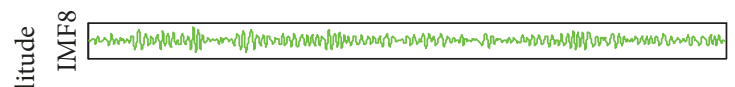

言
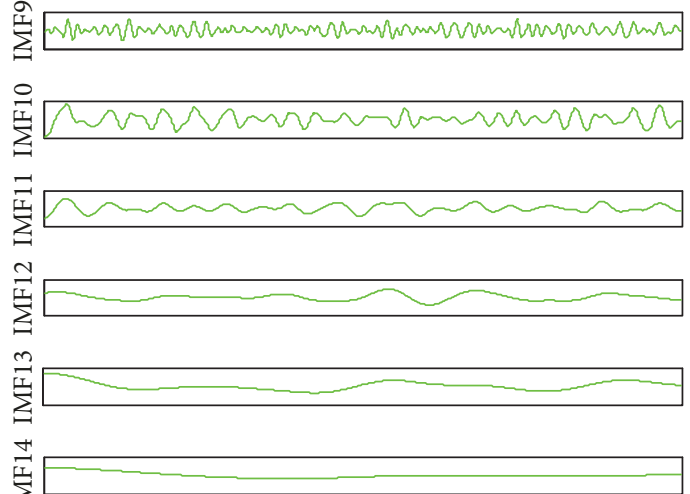

$\sum$
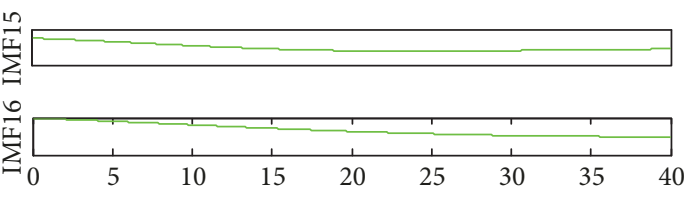

Time (s)

(b) EEMD

Figure 7: Signal decomposition of Stage (A). 


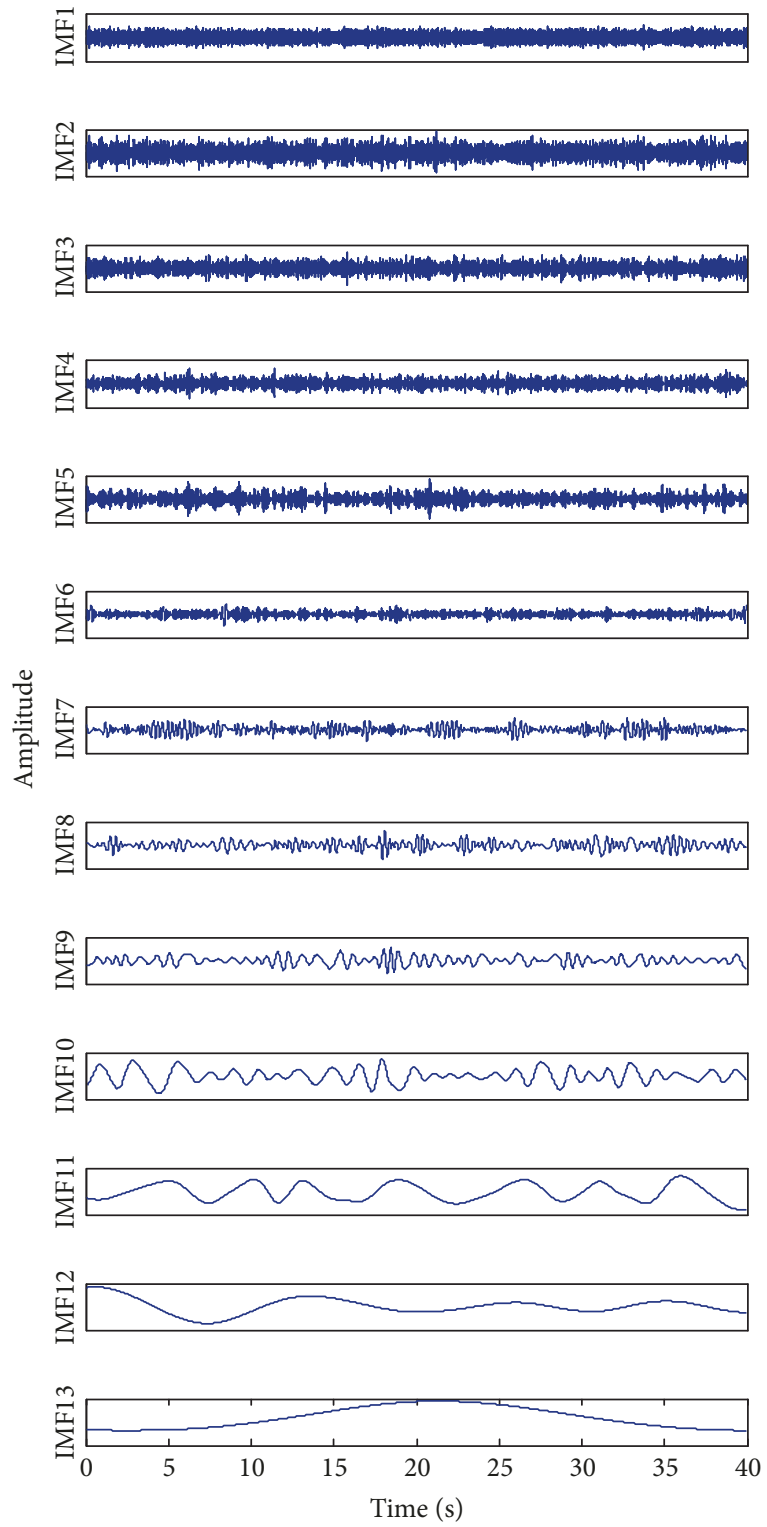

(a) CEEMD
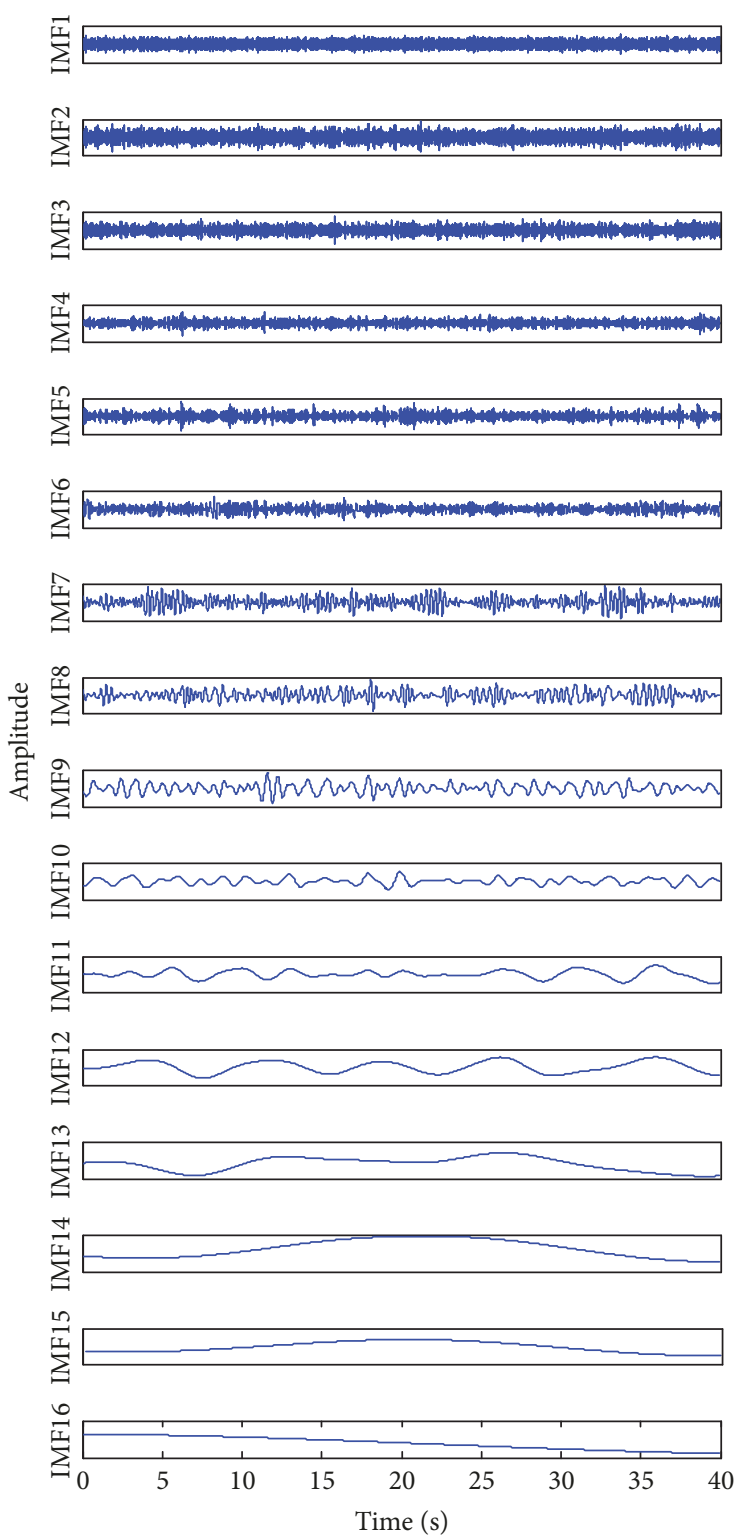

(b) EEMD

FIGURE 8: Signal decomposition of Stage (B).

The flow in Figure 5 shows the comparison among the 4 methods.

First, the direct-current components of the 3 original signals in Figure 2 were eliminated by removing the mean. The signals were predenoised using the MCKD algorithm. Figure 6 shows the time-domain signals after MCKD. Part of the background noise was eliminated, and the effects became more apparent.

The signals were then decomposed by CEEMD, and the results are shown in Figures 7(a), 8(a), and 9(a). For comparison, Figures 7(b), 8(b), and 9(b) show the EEMD results of the corresponding signals.

The time-domain signals are decomposed into a series of IMFs by CEEMD and EEMD. Although some of them contain plenty of failure feature components, others contain stronger background noise. Therefore, the IMFs should be selected before signal reconstruction, aiming to increase the SNR. In addition, the noise components are not to be considered. Kurtosis is a characteristic parameter that is sensitive to impact components. The value of kurtosis increases with the number of impact components in the signal. Correlation coefficient reflects the degree of correlation between the decomposed signals and the corresponding original signals. Therefore, on the condition of maintaining the basic features of the original signals, in order to extract fault features of slewing bearing from strong background noise, kurtosis and correlation coefficient are used to select IMFs to extract the fault features of the slewing bearing from strong background noise. The decomposition results of the vibration signals from the 3 stages are presented in Figures 10, 11, and 12 


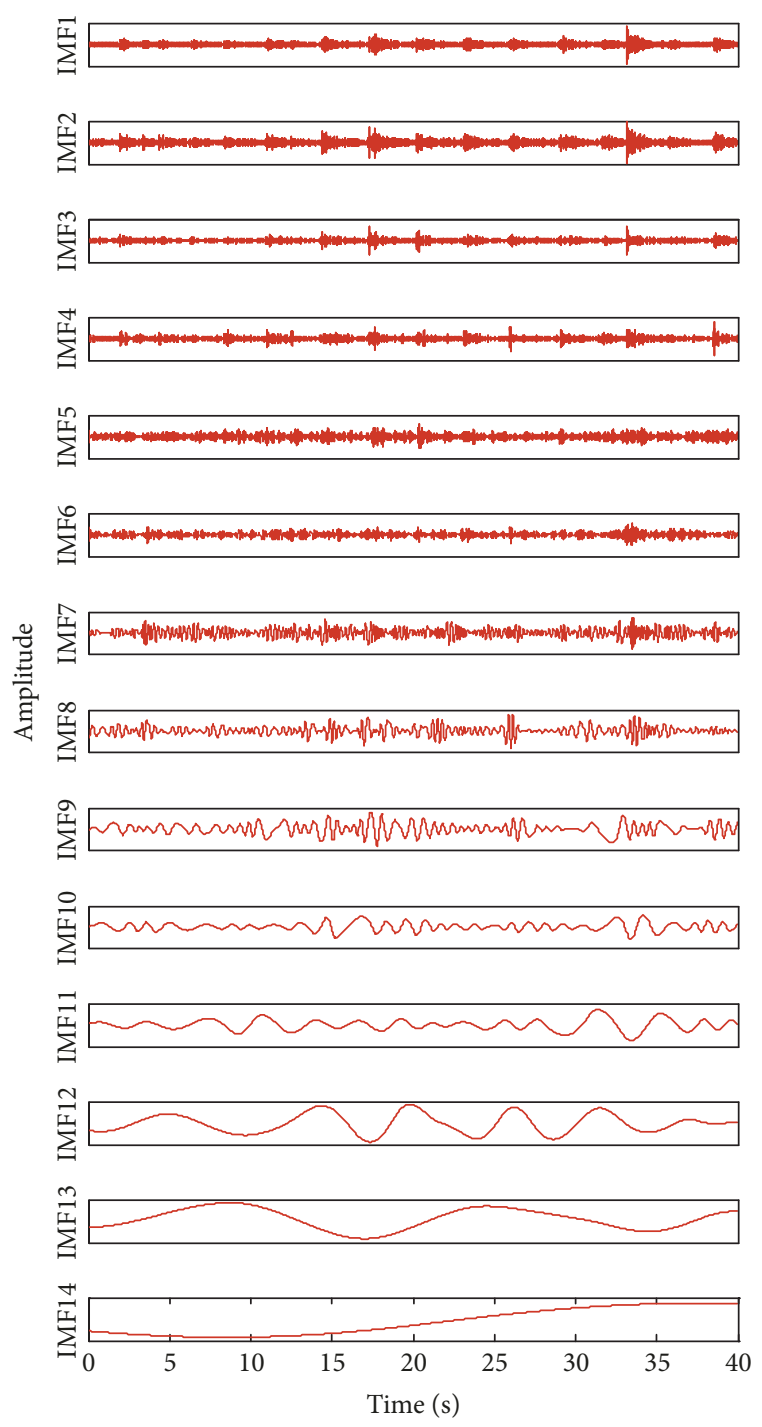

(a) CEEMD

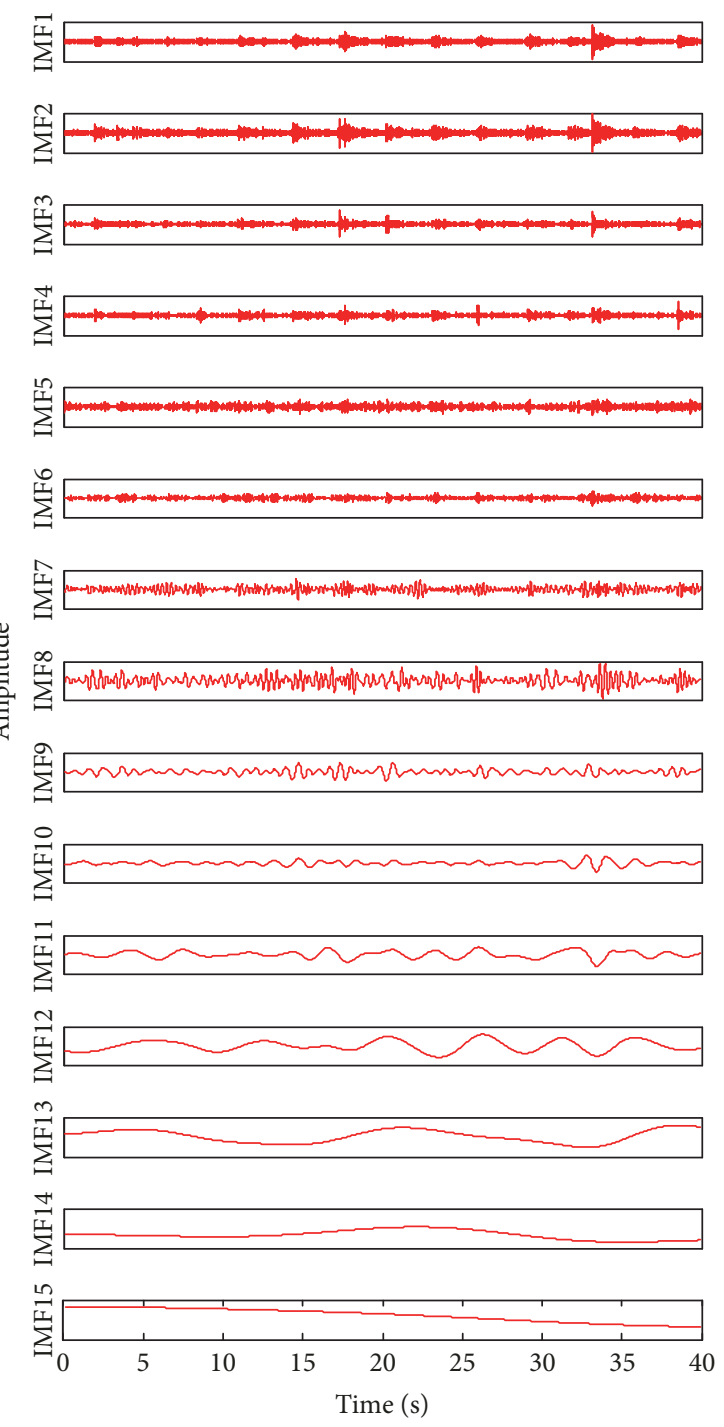

(b) EEMD

FIgURE 9: Signal decomposition of Stage (C).

TABLE 1: Ratio of parameter: kurtosis.

\begin{tabular}{|c|c|c|c|c|c|c|c|c|}
\hline \multirow{2}{*}{ Methods } & \multicolumn{7}{|c|}{ IMF $n$} & \multirow{2}{*}{ Stages } \\
\hline & 1 & 2 & 3 & 4 & 5 & 6 & 7 & \\
\hline \multicolumn{9}{|c|}{ Ratio (\%) } \\
\hline \multirow{3}{*}{ MCKD-CEEMD } & 5.16 & 12.1 & 19.5 & 27.5 & 35.0 & 42.5 & 50.8 & (A) \\
\hline & 5.28 & 13.2 & 21.2 & 29.9 & 38.8 & 48.7 & 57.7 & (B) \\
\hline & 11.9 & 24.1 & 40.2 & 53.1 & 58.8 & 64.8 & 69.5 & (C) \\
\hline \multirow{3}{*}{ MCKD-EEMD } & 4.36 & 10.3 & 16.6 & 23.5 & 29.5 & 36.3 & 45.8 & (A) \\
\hline & 4.74 & 11.7 & 19.0 & 27.5 & 36.1 & 43.2 & 51.9 & (B) \\
\hline & 11.0 & 22.5 & 39.0 & 51.9 & 56.6 & 61.5 & 66.0 & (C) \\
\hline \multirow{3}{*}{ CEEMD } & 4.93 & 11.4 & 18.2 & 28.4 & 36.1 & 45.3 & 52.2 & (A) \\
\hline & 4.27 & 10.1 & 35.7 & 43.4 & 49.4 & 56.0 & 65.5 & (B) \\
\hline & 12.7 & 27.1 & 43.3 & 49.2 & 53.7 & 59.8 & 64.9 & (C) \\
\hline
\end{tabular}




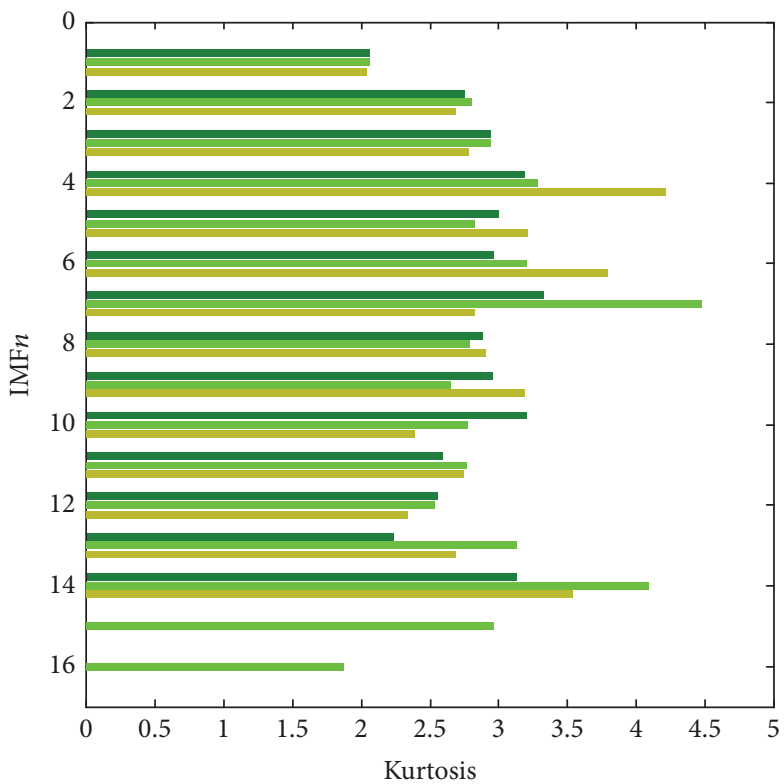

MCKD + CEEMD

MCKD + EEMD

CEEMD

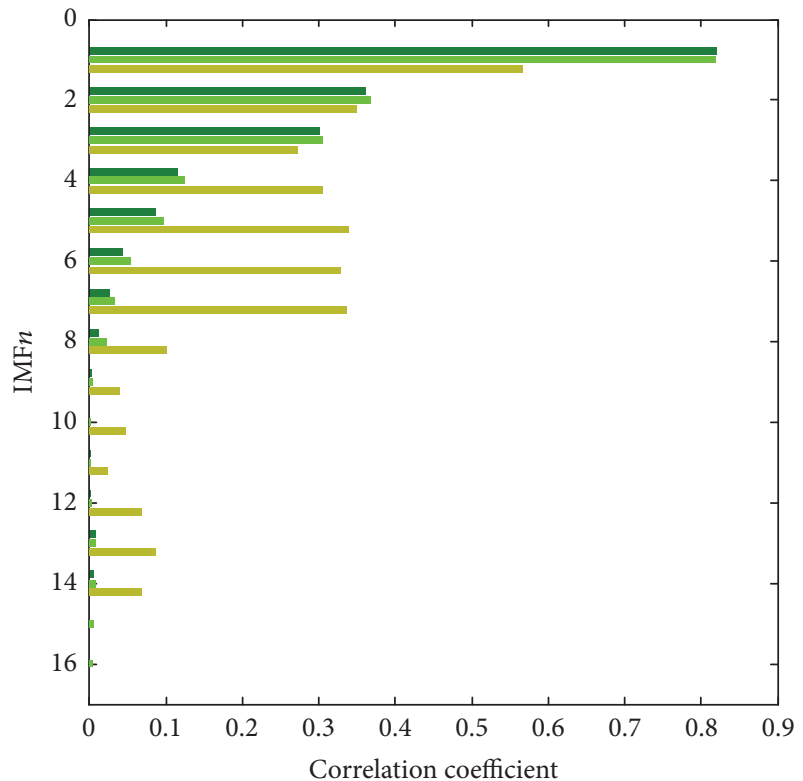

MCKD + CEEMD

MCKD + EEMD

CEEMD

(a) Kurtosis

(b) Correlation coefficient

FIgURE 10: Characteristic parameters of Stage (A).
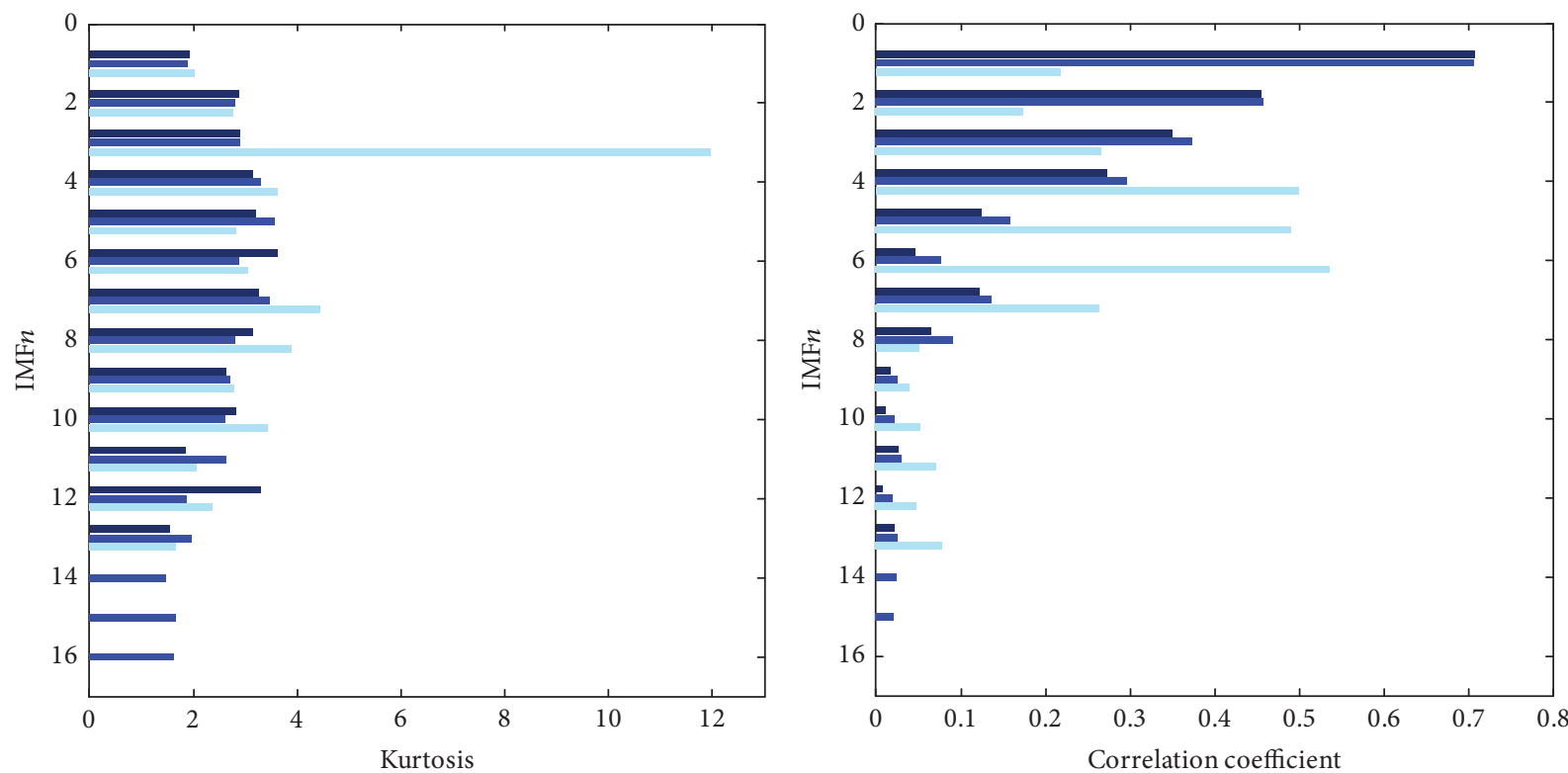

MCKD + CEEMD

MCKD + EEMD

CEEMD

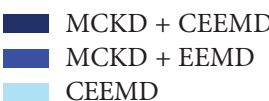

CEEMD
(a) Kurtosis
(b) Correlation coefficient

FIGURE 11: Characteristic parameters of Stage (B). 


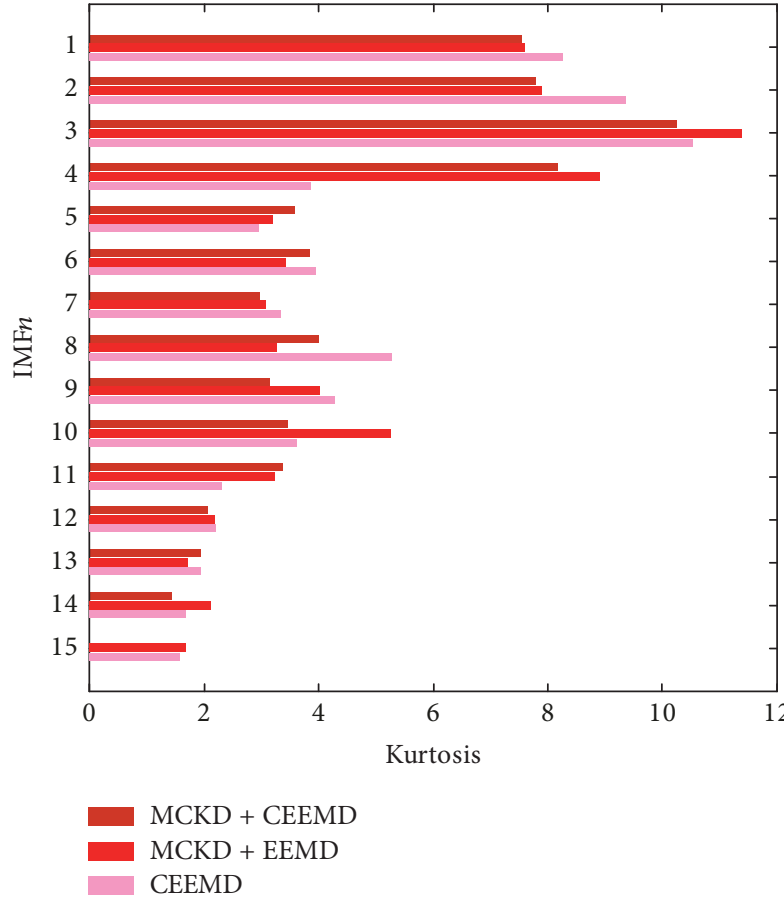

(a) Kurtosis

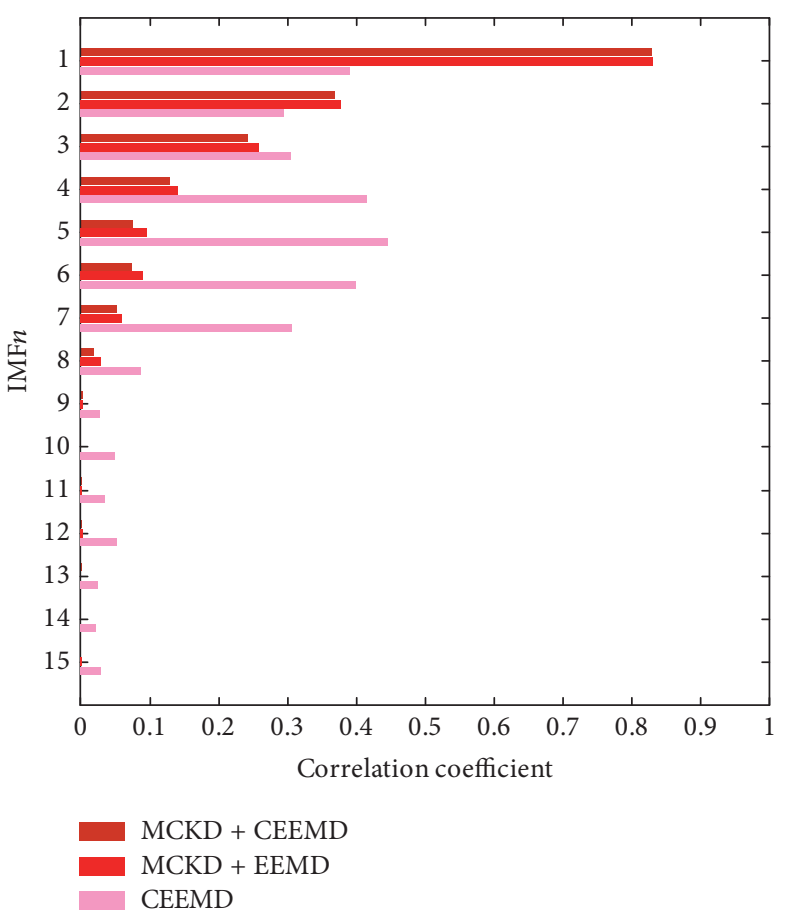

(b) Correlation coefficient

FIGURE 12: Characteristic parameters of Stage (C).

TABLE 2: Ratio of parameter: correlation coefficient.

\begin{tabular}{|c|c|c|c|c|c|c|c|c|}
\hline \multirow{2}{*}{ Methods } & \multicolumn{7}{|c|}{ IMF $n$} & \multirow{2}{*}{ Stages } \\
\hline & 1 & 2 & 3 & 4 & 5 & 6 & 7 & \\
\hline \multicolumn{9}{|c|}{ Ratio (\%) } \\
\hline \multirow{3}{*}{ MCKD-CEEMD } & 46.0 & 66.2 & 83.1 & 89.6 & 94.5 & 96.9 & 98.4 & (A) \\
\hline & 31.2 & 52.2 & 67.9 & 80.2 & 85.7 & 87.8 & 93.3 & (B) \\
\hline & 46.2 & 66.8 & 80.2 & 87.4 & 91.6 & 95.8 & 98.7 & (C) \\
\hline \multirow{3}{*}{ MCKD-EEMD } & 44.2 & 64.0 & 80.5 & 87.2 & 92.5 & 95.4 & 97.2 & (A) \\
\hline & 28.8 & 47.4 & 62.6 & 74.7 & 81.1 & 84.2 & 89.8 & (B) \\
\hline & 44.0 & 64.0 & 77.7 & 85.1 & 90.2 & 95.0 & 98.2 & $(\mathrm{C})$ \\
\hline \multirow{3}{*}{ CEEMD } & 19.4 & 31.3 & 40.6 & 50.9 & 62.5 & 73.7 & 85.2 & (A) \\
\hline & 7.82 & 14.0 & 23.6 & 41.5 & 59.1 & 78.4 & 87.9 & (B) \\
\hline & 13.5 & 23.8 & 34.4 & 48.8 & 64.2 & 78.1 & 88.7 & (C) \\
\hline
\end{tabular}

with different colors, representing MCKD-CEEMD, MCKDEEMD, and CEEMD, respectively.

The cumulative percentages of kurtosis and the correlation coefficient about the first $n$ IMFs of different signals comprising all of the corresponding features are shown in Tables 1 and 2. After the calculation of the first 7 IMFs, all cumulative percentages of the correlation coefficient values are not lower than $85 \%$, and the cumulative percentages are all not lower than 50\% (except for the result of MCKDEEMD in the normal stage). Therefore, the first 7 IMFs, which contain the majority of the principal characteristics and achieve denoising to a certain extent, are selected to represent the original signals of the corresponding stages.

The entropy features ApEn, SE, LLE, and CD values of the first 7 IMFs from different working stages, obtained by
TABLE 3: Results of ApEn of the proposed method.

\begin{tabular}{lccccccc}
\hline \multirow{2}{*}{ Stages } & & \multicolumn{7}{c}{ IMFn } \\
& 1 & 2 & 3 & 4 & 5 & 6 & 7 \\
\hline (A) & 0.447 & 0.251 & 0.172 & 0.089 & 0.058 & 0.046 & 0.032 \\
(B) & 0.598 & 0.393 & 0.288 & 0.202 & 0.150 & 0.113 & 0.085 \\
(C) & 0.771 & 0.450 & 0.350 & 0.173 & 0.179 & 0.171 & 0.134 \\
\hline
\end{tabular}

CEEMD or EEMD, are extracted. Table 3 shows the ApEn values of the 3 stages obtained using the proposed method. According to this idea, 25 one-rotation-cycle time-domain segments of each stage are selected to calculate ApEn, SE, LLE, and CD values, obtaining 3150 (75 signals $\times 7$ IMFs $\times$ ApEn/SE/LLE/CD values) values. 
TABLE 4: Data collection dates of the 150 segments.

\begin{tabular}{|c|c|c|c|c|c|c|c|c|c|c|}
\hline Stages & 1 & 2 & 3 & 4 & 5 & 6 & 7 & 8 & 9 & Notes \\
\hline \multirow{3}{*}{ (A) } & 150506 & 150625 & 150906 & 151015 & 151120 & 160122 & 160315 & 160420 & 160604 & Dates \\
\hline & 3 & 3 & 2 & 3 & 3 & 3 & 2 & 3 & 3 & Segments ${ }^{1}$ \\
\hline & 2 & 3 & 3 & 3 & 3 & 2 & 3 & 3 & 3 & Segments $^{2}$ \\
\hline \multirow{3}{*}{ (B) } & 160702 & 160915 & 161025 & 161210 & 170222 & 170411 & 170522 & 170618 & - & Dates \\
\hline & 2 & 3 & 3 & 4 & 4 & 3 & 3 & 3 & - & Segments $^{1}$ \\
\hline & 2 & 2 & 4 & 4 & 4 & 4 & 3 & 2 & - & Segments $^{2}$ \\
\hline \multirow{3}{*}{ (C) } & 170920 & 171026 & 171110 & 171228 & - & - & - & - & - & Dates \\
\hline & 6 & 6 & 6 & 7 & - & - & - & - & - & Segments ${ }^{1}$ \\
\hline & 5 & 7 & 8 & 5 & - & - & - & - & - & Segments $^{2}$ \\
\hline
\end{tabular}

${ }^{1} 75$ segments for feature extraction; ${ }^{2} 75$ segments for condition classification.

TABLE 5: Classification results.

\begin{tabular}{|c|c|c|c|c|}
\hline Labels & Label 1 & Label 2 & Label 3 & Classification \\
\hline Samples & 25 & 25 & 25 & rate $(\%)$ \\
\hline \multirow{3}{*}{$\begin{array}{l}\text { MCKD-CEEMD- } \\
\text { ApEn }\end{array}$} & 23 & 2 & & \multirow{3}{*}{93.3} \\
\hline & 2 & 23 & & \\
\hline & & 1 & 24 & \\
\hline \multirow{3}{*}{ MCKD-CEEMD-LLE } & 23 & 1 & 1 & \multirow{3}{*}{89.3} \\
\hline & 1 & 22 & 2 & \\
\hline & & 3 & 22 & \\
\hline \multirow{3}{*}{ MCKD-CEEMD-CD } & 24 & & 1 & \multirow{3}{*}{92.0} \\
\hline & 1 & 22 & 2 & \\
\hline & 2 & & 23 & \\
\hline \multirow{3}{*}{ MCKD-CEEMD-SE } & 23 & 2 & & \multirow{3}{*}{88.0} \\
\hline & 3 & 21 & 1 & \\
\hline & 1 & 2 & 22 & \\
\hline \multirow{3}{*}{ MCKD-EEMD-ApEn } & 22 & 3 & & \multirow{3}{*}{89.3} \\
\hline & 3 & 22 & & \\
\hline & & 2 & 23 & \\
\hline \multirow{3}{*}{ CEEMD-ApEn } & 20 & 5 & & \multirow{3}{*}{85.3} \\
\hline & 3 & 21 & 1 & \\
\hline & & 2 & 23 & \\
\hline
\end{tabular}

The 3150 values are inputted into SVM for training. In addition, 3150 values of 75 additional signals from 3 different working stages were calculated using the same methods. The overall data collection dates of the 150 segments are shown in Table 4. The latter 3150 values are used to verify the classification results of the SVM, which are listed in Table 5, where labels 1,2 , and 3 represent the normal condition, early fault condition, and severe fault condition, respectively. Compared with the 3 control methods, the proposed methods obtain the highest classification rate. Besides, ApEn could be taken as a better characteristic parameter than LLE and CD in fault diagnosis and classification of slewing bearings. With the practical working environment of the slewing bearing and the complexity of the port crane considered, the noise interference in vibration signals is much stronger than the noise interference collected from simulant test platforms. Therefore, although the $100 \%$ classification rate is rather difficult to obtain, the proposed method and the results can also provide a great value for reference in practical engineering.

\section{Conclusions}

This study proposed a combined failure diagnosis method based on MCKD-CEEMD-ApEn for large-size and heavyload slewing bearings. The original signals were denoised by removing the mean, MCKD algorithm, and CEEMD to obtain the first 7 IMFs, which could maintain the features of original signals and remove part of the strong background noise. In this method, ApEn was adopted as the classification feature and SVM was used to verify the reliable classification rate. Owing to the greater interest drawn by the severe fault stage compared with the early fault stage of the slewing bearings in practical engineering, the methods considered the features of the 3 conditions and conducted method comparison and classification, which could be widely used in a wide range of applications in fault diagnosis and could provide a method to distinguish various working conditions of slewing bearings.

\section{Data Availability}

The data used to support the findings of this study are available from the corresponding author upon request.

\section{Conflicts of Interest}

The authors declare that there are no conflicts of interest regarding the publication of this paper.

\section{Acknowledgments}

This paper is supported by the scientific project (no. KJ (Y) 2015013) of Jiangsu Province Special Equipment Safety Supervision Inspection Institute, Branch of Wuxi, and the National Natural Science Foundation of China (no. 51375067). 


\section{References}

[1] F. Wang, C. Liu, W. Su, Z. Xue, H. Li, and Q. Han, "Condition monitoring and fault diagnosis methods for low-speed and heavy-load slewing bearings: a literature review," Journal of Vibroengineering, vol. 19, no. 5, pp. 3429-3444, 2017.

[2] C. Mishra, A. K. Samantaray, and G. Chakraborty, "Rolling element bearing fault diagnosis under slow speed operation using wavelet de-noising," Measurement, vol. 103, pp. 77-86, 2017.

[3] M. Žvokelj, S. Zupan, and I. Prebil, "EEMD-based multiscale ICA method for slewing bearing fault detection and diagnosis," Journal of Sound and Vibration, vol. 370, pp. 394-423, 2016.

[4] J. Yang, J. Chen, R. Hong, H. Wang, and Y. Feng, "Research of slew bearing signal de-noising based on multi-scale principal component analysis and EEMD," Zhongnan Daxue Xuebao (Ziran Kexue Ban)/Journal of Central South University (Science and Technology), vol. 47, no. 4, pp. 1173-1180, 2016.

[5] W. Caesarendra, P. B. Kosasih, A. K. Tieu, C. A. S. Moodie, and B.-K. Choi, "Condition monitoring of naturally damaged slow speed slewing bearing based on ensemble empirical mode decomposition," Journal of Mechanical Science and Technology, vol. 27, no. 8, pp. 2253-2262, 2013.

[6] G. L. McDonald, Q. Zhao, and M. J. Zuo, "Maximum correlated Kurtosis deconvolution and application on gear tooth chip fault detection," Mechanical Systems and Signal Processing, vol. 33, pp. 237-255, 2012.

[7] N. Sawalhi, R. B. Randall, and H. Endo, "The enhancement of fault detection and diagnosis in rolling element bearings using minimum entropy deconvolution combined with spectral kurtosis," Mechanical Systems and Signal Processing, vol. 21, no. 6, pp. 2616-2633, 2007.

[8] F. Jia, Y. Lei, H. Shan, and J. Lin, "Early fault diagnosis of bearings using an improved spectral kurtosis by maximum correlated kurtosis deconvolution," Sensors, vol. 15, no. 11, pp. 29363-29377, 2015.

[9] H. Zhao and L. Li, "Incipient bearing fault diagnosis based on MCKD-EMD for wind turbine," Dianli Zidonghua Shebei/Electric Power Automation Equipment, vol. 37, no. 2, pp. 2936, 2017.

[10] J.-R. Yeh, J.-S. Shieh, and N. E. Huang, "Complementary ensemble empirical mode decomposition: a novel noise enhanced data analysis method," Advances in Adaptive Data Analysis: Theory and Applications, vol. 2, no. 2, pp. 135-156, 2010.

[11] D.-H. Lee, J.-H. Ahn, and B.-H. Koh, "Fault detection of bearing systems through EEMD and optimization algorithm," Sensors, vol. 17, no. 11, Article ID 2477, 2017.

[12] Y. Imaouchen, M. Kedadouche, R. Alkama, and M. Thomas, "A frequency-weighted energy operator and complementary ensemble empirical mode decomposition for bearing fault detection," Mechanical Systems and Signal Processing, vol. 82, pp. 103-116, 2017.

[13] X. Xue, J. Zhou, Y. Xu, W. Zhu, and C. Li, "An adaptively fast ensemble empirical mode decomposition method and its applications to rolling element bearing fault diagnosis," Mechanical Systems and Signal Processing, vol. 62, pp. 444-459, 2015.

[14] M. J. Li, H. Q. Wang, G. Tang, H. F. Yuan, and Y. Yang, "An improved method based on CEEMD for fault diagnosis of rolling bearing," Advances in Mechanical Engineering, vol. 6, Article ID 676205, 10 pages, 2014.
[15] A. Rabah and K. Abdelhafid, "Rolling bearing fault diagnosis based on improved complete ensemble empirical mode of decomposition with adaptive noise combined with minimum entropy deconvolution," Journal of Vibroengineering, vol. 20, no. 1, pp. 240-257, 2018.

[16] J. Zheng, H. Pan, S. Yang, and J. Cheng, "Generalized composite multiscale permutation entropy and Laplacian score based rolling bearing fault diagnosis," Mechanical Systems and Signal Processing, vol. 99, pp. 229-243, 2018.

[17] S. Tong, J. Xu, F. Cong, N. Tang, and Y. Zhang, "Fault diagnosis method based on the slip information entropy and optimal filter construction," Journal of Vibration and Shock, vol. 36, pp. 34-39, 2017.

[18] M. Lei, G. Meng, and G. Dong, "Fault detection for vibration signals on rolling bearings based on the symplectic entropy method," Entropy, vol. 19, no. 11, Article ID 607, 2017.

[19] S. M. Pincus, "Approximate entropy as a measure of system complexity," Proceedings of the National Acadamy of Sciences of the United States of America, vol. 88, no. 6, pp. 2297-2301, 1991.

[20] X. An and L. Pan, "Wind turbine bearing fault diagnosis based on adaptive local iterative filtering and approximate entropy," Proceedings of the Institution of Mechanical Engineers, Part C: Journal of Mechanical Engineering Science, vol. 231, no. 17, pp. 3228-3237, 2017.

[21] W. Caesarendra and T. Tjahjowidodo, "A review of feature extraction methods in vibration-based condition monitoring and its application for degradation trend estimation of lowspeed slew bearing," Machines, vol. 5, no. 4, Article ID 21, 2017.

[22] K. Li, L. Su, J. Wu, H. Wang, and P. Chen, "A rolling bearing fault diagnosis method based on variational mode decomposition and an improved kernel extreme learning machine," Applied Sciences (Switzerland), vol. 7, no. 10, Article ID 1004, 2017.

[23] W. Caesarendra, B. Kosasih, K. Tieu, and C. A. S. Moodie, "An application of nonlinear feature extraction-A case study for low speed slewing bearing condition monitoring and prognosis," in Proceedings of the 2013 IEEE/ASME International Conference on Advanced Intelligent Mechatronics: Mechatronics for Human Wellbeing, AIM 2013, pp. 1713-1718, Australia, July 2013.

[24] V. N. Vapnik, Statistical Learning Theory, Wiley- Interscience, New York, NY, USA, 1998.

[25] J. D. Martínez-Morales, E. R. Palacios-Hernández, and D. U. Campos-Delgado, "Multiple-fault diagnosis in induction motors through support vector machine classification at variable operating conditions," Electrical Engineering, vol. 100, no. 1, pp. 59-73, 2018.

[26] G. L. Xiong, L. Zhang, H. S. Liu, H. J. Zou, and W.-Z. Guo, "A comparative study on ApEn, SampEn and their fuzzy counterparts in a multiscale framework for feature extraction," Journal of Zhejiang University SCIENCE A, vol. 11, no. 4, pp. 270279, 2010.

[27] X. L. Zhang, X. F. Chen, and Z. J. He, "Fault diagnosis based on support vector machines with parameter optimization by an ant colony algorithm," Proceedings of the Institution of Mechanical Engineers, Part C: Journal of Mechanical Engineering Science, vol. 224, no. 1, pp. 217-229, 2010.

[28] S. Liu, G. Tang, and Y. He, "Incipient fault diagnosis of rolling bearings based on Teager energy operator and MCKD," Journal of Vibration and Shock, vol. 35, no. 15, pp. 98-102, 2016.

[29] N. E. Huang and Z. Wu, "A review on Hilbert-Huang transform: method and its applications to geophysical studies," Reviews of Geophysics, vol. 46, no. 2, Article ID RG2006, 2008. 


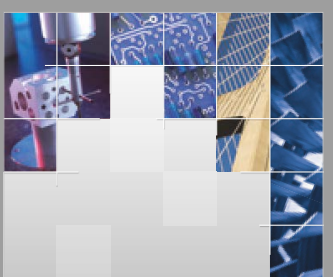

\section{Enfincering}
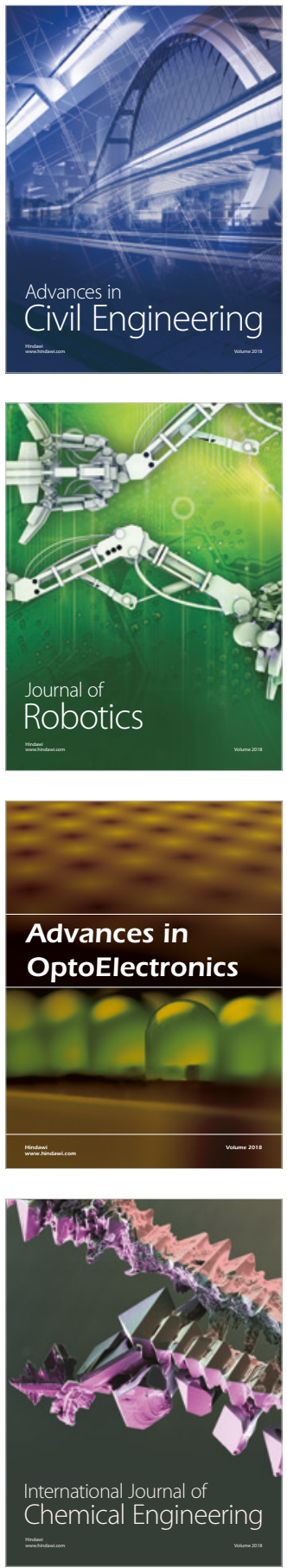

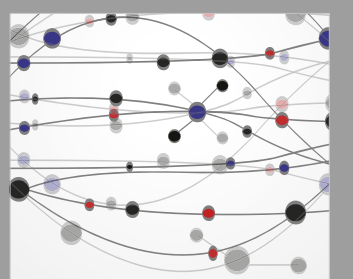

\section{Rotating \\ Machinery}

The Scientific World Journal

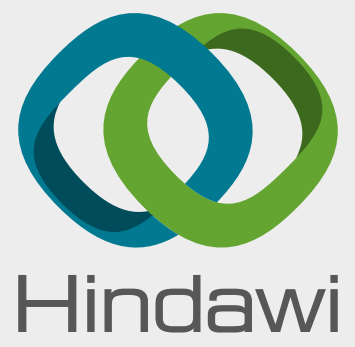

Submit your manuscripts at

www.hindawi.com
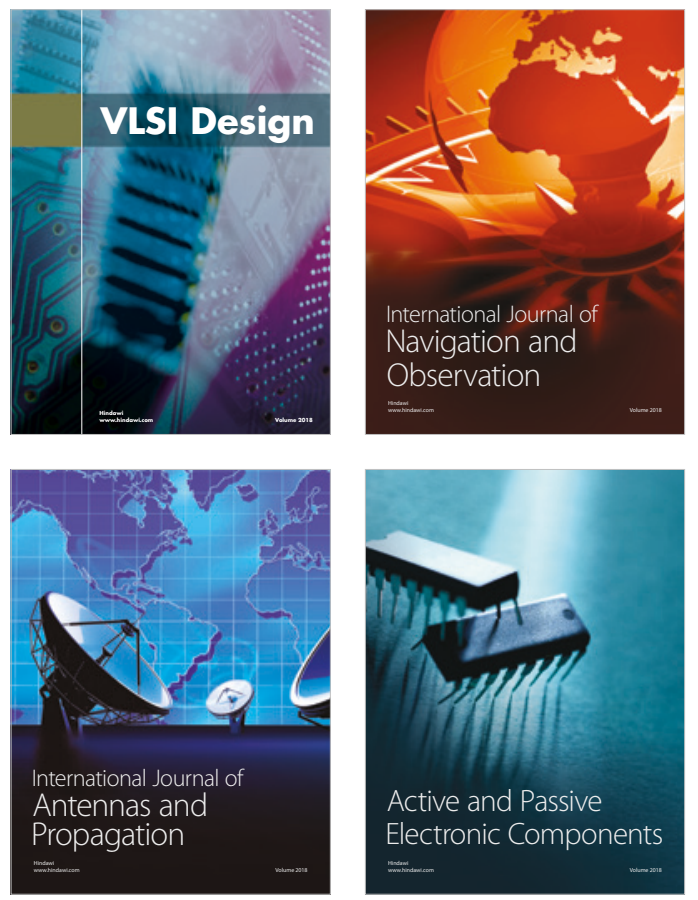
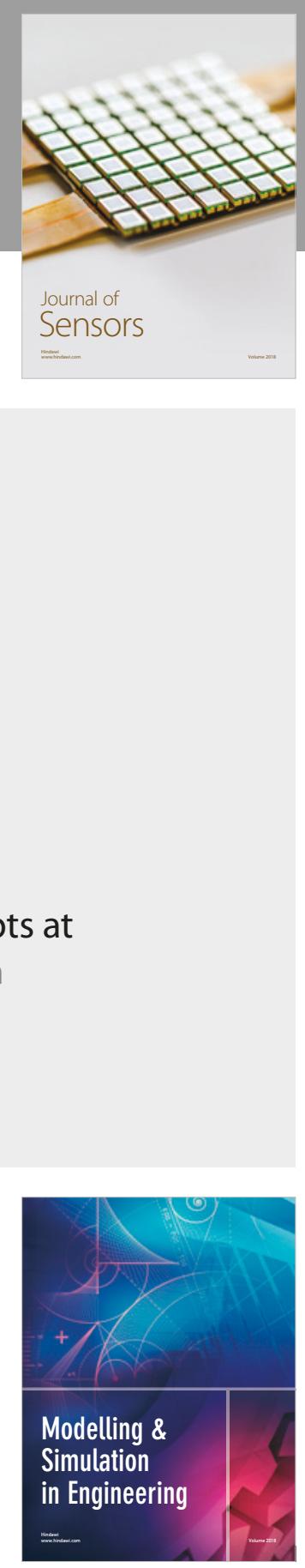

\section{Advances \\ Multimedia}
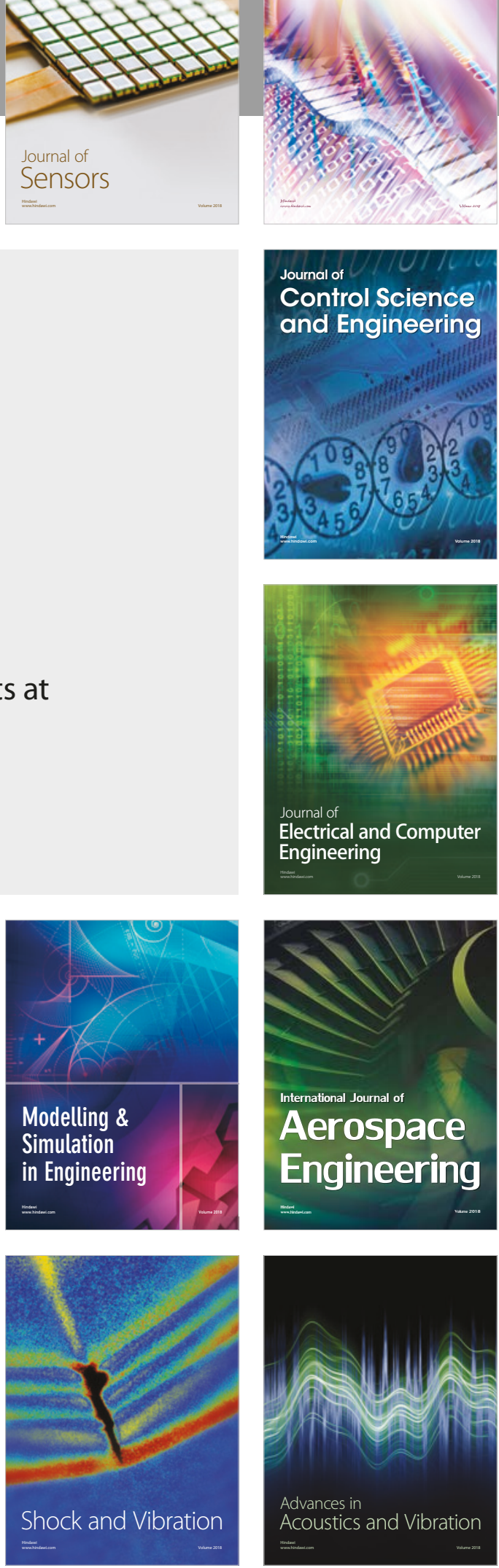